\title{
Transcriptional analysis distinguishes breast implant-associated anaplastic large cell lymphoma from other peripheral T-cell lymphomas
}

\author{
Arianna Di Napoli $\mathbb{D}^{1} \cdot$ Loris De Cecco ${ }^{2}$ Pier Paolo Piccaluga $\mathbb{1}^{3,4} \cdot$ Mohsen Navari $^{5} \cdot$ Valeria Cancila $^{6}$. \\ Claudia Cippitelli ${ }^{1}$ - Giuseppina Pepe ${ }^{1}$. Gianluca Lopez ${ }^{1}$. Francesca Monardo ${ }^{7}$. Antonella Bianchi ${ }^{8}$. \\ Emanuele Stefano Giovanni D'Amore ${ }^{9}$. Umberto Gianelli ${ }^{10} \cdot$ Fabio Facchetti $^{11} \cdot$ Emilio Berti $^{12} \cdot$ Govind Bhagat $^{13}$
}

Received: 22 April 2018 / Revised: 6 August 2018 / Accepted: 7 August 2018 / Published online: 11 September 2018

(c) United States \& Canadian Academy of Pathology 2018

\begin{abstract}
Breast implant-associated anaplastic large cell lymphoma is a new provisional entity in the revised World Health Organization classification of lymphoid malignancies, the pathogenesis and cell of origin of which are still unknown. We performed gene expression profiling of microdissected breast implant-associated anaplastic large cell lymphoma samples and compared their transcriptional profiles with those previously obtained from normal T-cells and other peripheral T-cell lymphomas and validated expression of selected markers by immunohistochemistry. Our results indicate that most breast implant-associated anaplastic large cell lymphomas exhibit an activated CD4+ memory T-cell phenotype, which is associated with CD25 and FoxP3 expression. Gene ontology analyses revealed upregulation of genes involved in cell motility programs (e.g., CCR6, MET, HGF, CXCL14) in breast implant-associated anaplastic large cell lymphomas compared to normal CD4+ T-cells and upregulation of genes involved in myeloid cell differentiation (e.g., PPARg, JAK2, SPI-1, GAB2) and viral gene transcription (e.g., RPS10, RPL17, RPS29, RPL18A) compared to other types of peripheral T-cell lymphomas. Gene set enrichment analyses also revealed shared features between the molecular profiles of breast implant-associated anaplastic large cell lymphomas and other types of anaplastic large cell lymphomas, including downregulation of T-cell receptor signaling and STAT3 activation. Our findings
\end{abstract}

Electronic supplementary material The online version of this article (https://doi.org/10.1038/s41379-018-0130-7) contains supplementary material, which is available to authorized users.

$\triangle$ Arianna Di Napoli

arianna.dinapoli@uniroma1.it

$\triangle$ Govind Bhagat

gb96@cumc.columbia.edu

1 Department of Clinical and Molecular Medicine, Sapienza University of Rome, Sant'Andrea Hospital, Rome, Italy

2 Integrated Biology Platform, Department of Applied Research and Technology Development, Fondazione IRCCS Istituto Nazionale dei Tumori, Milan, Italy

3 Department of Experimental, Diagnostic and Experimental Medicine, Bologna University School of Medicine, Bologna, Italy

4 Euro-Mediterranean Institute of Science and Technology (IEMEST), 90139 Palermo, Italy

5 Research Center of Advanced Technologies in Medicine, Torbat Heydariyeh University of Medical Sciences, Torbat Heydariyeh, Iran

6 Tumor Immunology Unit, Dipartimento per la Promozione della provide novel insights into the biology of this rare disease and further evidence that breast implant-associated anaplastic large cell lymphoma represents a distinct peripheral T-cell lymphoma entity.

Salute e Materno Infantile “G. D'Alessandro”, University of Palermo, Palermo, Italy

7 Pathology Unit, San Giovanni Addolorata Hospital, Roma, Italy

8 Pathology Unit, University Hospital Campus Bio-Medico, Rome, Italy

9 Institute of Pathology, San Bortolo Hospital, Vicenza, Italy

10 Hematopathology Unit, Department of Physiopathology and Transplantation, IRCCS Ca' Granda Ospedale Maggiore Policlinico, University of Milan, Milan, Italy

11 Pathology Section, Department of Molecular and Translational Medicine, University-Spedali Civili of Brescia, Spedali Civili di Brescia, Brescia, Italy

12 Dermatology Unit, Department of Physiopathology and Transplantation, IRCCS Ca' Granda Ospedale Maggiore Policlinico, University of Milan, Milan, Italy

13 Department of Pathology and Cell Biology, Columbia University Medical Center, New York Presbyterian Hospital, New York, NY, USA 


\section{Introduction}

Breast implant-associated anaplastic large cell lymphoma is a rare malignancy that arises around mammary prosthetic implants in patients who have undergone breast augmentation or reconstruction [1-3]. In the recent revision of the World Health Organization classification of lymphoid neoplasms, breast implant-associated anaplastic large cell lymphoma has been included as a provisional entity [4].

Anaplastic large cell lymphomas comprise different entities (i.e., systemic, primary cutaneous, breast implantassociated), which despite sharing the presence of CD30positive large atypical lymphocytes, substantially differ in their clinical presentation and behavior [4-8]. Among systemic anaplastic large cell lymphomas, those harboring translocations of the anaplastic lymphoma kinase gene $(A L K)$ (ALK-positive anaplastic large cell lymphoma) have a more favorable clinical course compared to those lacking this genetic lesion (ALK-negative anaplastic large cell lymphoma) [6, 7]. Breast implant-associated anaplastic large cell lymphoma has an ALK-negative phenotype and cases confined to the peri-implant breast seroma, without invasion of the fibrous capsule, have excellent outcomes [5], similar to primary cutaneous anaplastic large cell lymphomas $[7,8]$.

The pathogenesis of breast implant-associated anaplastic large cell lymphoma remains elusive. Chronic stimulation of the immune system elicited by prosthetic materials and biofilms, perhaps in genetically predisposed individuals, has been hypothesized to have a role in disease pathogenesis [9]. However, in contrast to other forms of ALK-negative anaplastic large cell lymphomas, the molecular features underlying breast implant-associated anaplastic large cell lymphoma pathobiology are largely unknown, thus limiting the identification of biomarkers that could assist in diagnosis, prognosis and appropriate therapeutic intervention.

Gene expression profiling studies have revealed distinct but also common features among anaplastic large cell lymphomas, independent of ALK status, implying shared pathways of lymphomagenesis [10, 11]. Indeed, identification of a set of 30 differentially expressed genes in both ALK-positive and ALK-negative anaplastic large cell lymphomas compared to other types of peripheral T-cell lymphomas suggested the existence of a common anaplastic large cell lymphoma signature, including the loss of T-cell receptor signaling [11]. Different classifiers have been proposed to distinguish ALK-negative anaplastic large cell lymphoma from peripheral T-cell lymphoma, not otherwise specified, including CD30+ cases $[11,12]$. However, it has been shown that CD30+ peripheral T-cell lymphomas, not otherwise specified, and ALK-negative anaplastic large cell lymphomas share an expression signature that is distinct from that of $\mathrm{CD} 30$ - peripheral T-cell lymphomas, not otherwise specified, which is characterized by the reduced expression of T-cell receptor-associated proximal tyrosine kinases (LCK, FYN, ITK) and molecules regulating T-cell differentiation/activation (CD69, ICOS, CD52, NFATc2), as well as increased JUNB and IRF4 expression [13, 14].

To gain insights into the transcriptional landscape of breast implant-associated anaplastic large cell lymphoma and its relationship with other types of peripheral T-cell lymphomas, we performed gene expression profiling of microdissected tumor samples of breast implant-associated anaplastic large cell lymphoma and compared the expression profiles with those reported for other types of anaplastic large cell lymphomas, angioimmunoblastic T-cell lymphoma and peripheral T-cell lymphomas, not otherwise specified.

\section{Methods}

\section{Sample collection}

Gene expression and immunohistochemical analyses of six breast implant-associated anaplastic large cell lymphoma cases were performed, which were collected from the Sant'Andrea Hospital of Rome, Spedali Civili of Brescia, and $\mathrm{Ca}$ ' Granda Hospital of Milan (Italy). The clinicopathological characteristics of these breast implantassociated anaplastic large cell lymphomas were detailed previously $[1,2,5,15,16]$. Another six breast implantassociated anaplastic large cell lymphoma cases were obtained from the Sant'Andrea Hospital, San Giovanni Hospital and Campus Biomedico in Rome, San Bortolo Hospital in Vicenza, and Columbia University Medical Center in New York (USA) for validation analyses. Detailed pathological features of all breast implantassociated anaplastic large cell lymphomas are summarized in Table 1. In addition, four cases each of ALKpositive anaplastic large cell lymphoma, ALK-negative anaplastic large cell lymphoma, and peripheral T-cell lymphoma, not otherwise specified, diagnosed at the Sant'Andrea Hospital, Rome, and 16 cases of cutaneous anaplastic large cell lymphoma, diagnosed at Ca' Granda Hospital, Milan were used as controls for mRNA- or protein-based validation assays. The study was approved by the Ethics Committee of Sant'Andrea Hospital/University "Sapienza" of Rome (EC n. 82 SA_2017) and research was performed in accordance with the Declaration of Helsinki.

\section{Laser microdissection and RNA extraction}

Many breast implant-associated anaplastic large cell lymphoma samples have necrosis and a limited number of viable cells and the tumor cells are often adherent to the 


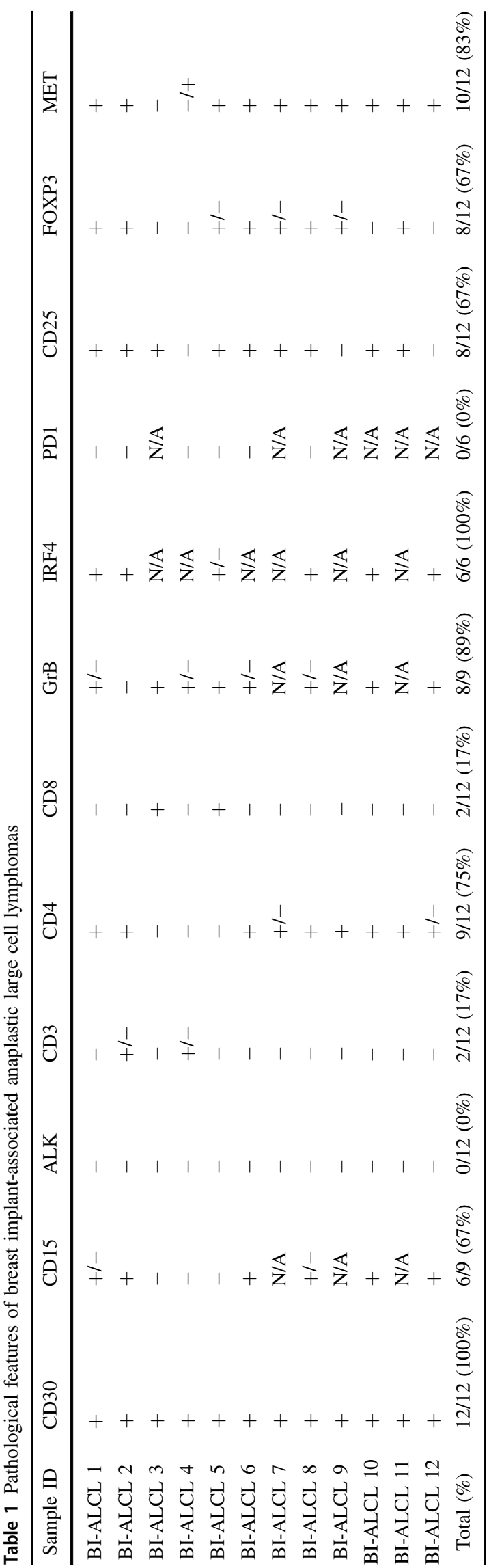

surface of the fibrous capsule and/or partially infiltrate it along with other inflammatory cells. Hence, to enrich for tumor cells and minimize contamination by RNA derived from non-neoplastic stromal and inflammatory cells, we performed laser microdissection of formalin-fixed paraffin embedded tissue sections of breast implantassociated anaplastic large cell lymphomas using the Laser Microdissector SL CUT (Nikon Instruments, New York, USA) as previously described [17]. Total RNA was then extracted and purified using High Pure miRNA Isolation Kit (Roche, Basel, Switzerland) from a total of $\sim 3000$ microdissected tumor cells per breast implant-associated anaplastic large cell lymphoma (Supplementary Figure 1) and from whole-tissue sections of peripheral T-cell lymphoma, not otherwise specified, cutaneous anaplastic large cell lymphoma and systemic ALK-negative and ALKpositive anaplastic large cell lymphoma samples with high tumor content, according to the manufacturer's instructions.

\section{Gene expression analysis}

RNA extracted from six microdissected breast implantassociated anaplastic large cell lymphoma cases was processed using the Whole-Genome DASL assay and hybridized on HumanHT-12 v4 Expression BeadChips (Illumina, San Diego, CA, USA) according to the vendor's instructions.

Gene expression profile analysis of six breast implantassociated anaplastic large cell lymphomas was performed and the profiles were compared with those generated previously for 10 samples of purified normal CD4+ T cells, 6 ALK-positive anaplastic large cell lymphomas, 6 ALKnegative anaplastic large cell lymphomas, 10 angioimmunoblastic T-cell lymphomas, and 80 peripheral T-cell lymphomas, not otherwise specified, including $18 \mathrm{CD} 30+$ peripheral T-cell lymphomas, not otherwise specified (i.e., CD30 antigen being expressed by $\geq 50 \%$ of neoplastic cells) [12]. Gene expression analysis was carried out according to MIAME guidelines. The data of each array were normalized using the "limma" package of Bioconductor [18] in $\mathrm{R}$ version 3.3.1. Background correction was inferred from detection of $p$-values and subtracted from the data, and the data were further quantile-normalized. Since the data were generated using different versions of the DASL array, common probes were selected for analysis. To remove batch effects, we used the ComBat package [19] in R version 3.3.1 as previously described [20]. Mean-variance normalization was performed using geWorkbench software (Supplementary Figure 2) [21].

Unsupervised and supervised analysis (including principal component analysis and hierarchical clustering) and assessment of differentially expressed genes was carried out using GeneSpring GX 12 (Agilent, USA), as previously described [12]. When identifying differentially expressed 
genes, by either $t$-test or one-way ANOVA, genes with at least twofold change in expression level and a $p$-value $<$ 0.05 were chosen for supervised analysis. Genes associated with RPS10 were identified using Pearson correlation (i.e., those with correlation $>0.5$ ).

Gene Ontology Consortium Panther Classification System was used for identifying the enrichment of signatures in differentially expressed genes (http://geneontology.org/pa ge/go-enrichment-analysis). Gene set enrichment analysis software of the Broad Institute (http://software.broa dinstitute.org/gsea/index.jsp) was employed to assess significant enrichment of Hallmark gene sets, considering those with False Discovery Rate $<0.05$.

Cell type deconvolution was performed using the CIBERSORT algorithm [22].

\section{Real-time polymerase chain reaction analysis}

Real-time quantitative polymerase chain reaction was performed with a Rotor-Gene Q system (Qiagen) using the Quantinova Probe RT-PCR kit (Qiagen) according to the manufacturer's instructions. Parallel amplification reactions were carried out using TaqMan gene expression assays with the RPS10 probe Hs01652370_gH and ACTB probe Hs01060665_g1. The polymerase chain reaction cycling conditions were as follows: $45^{\circ} \mathrm{C}$ for $10 \mathrm{~min}, 95^{\circ} \mathrm{C}$ for 5 min, followed by 40 cycles at $95^{\circ} \mathrm{C}$ for $5 \mathrm{~s}$ and $60^{\circ} \mathrm{C}$ for 30 s. Assays were performed in duplicate instead of triplicate due to the low amount of RNA obtained from microdissected samples. Gene expression results for RPS10 were normalized to $A C T B$ expressions and calculated using the $\Delta \Delta \mathrm{Ct}$ method as previously described [23]. Results were expressed as relative levels of RPS10 mRNA in different peripheral T-cell lymphoma samples ( 9 breast implantassociated anaplastic large cell lymphomas, 16 cutaneous anaplastic large cell lymphomas, 4 ALK-positive anaplastic large cell lymphomas, 4 ALK-negative anaplastic large cell lymphomas, 4 peripheral T-cell lymphomas, not otherwise specified) compared to RPS10 mRNA of a breast parenchyma sample that was chosen to represent $1 \times$ expression. The breast tissue sample was chosen as an external reference based on the mRNA expression profiles of normal tissues reported by The Human Protein Atlas (http://www. proteinatlas.org/ENSG00000124614-RPS10/tissue). The expression levels of RPS10 mRNA among the different samples were compared using the Student's $t$-test.

\section{Immunohistochemistry}

Immunohistochemistry for T-cell antigens, IRF4, PD1, LMP-1, and KSHV/LANA-1 was performed on formalinfixed paraffin embedded tissue sections at different centers according to standard methods. Immunohistochemistry for
RPS10 (clone EPR8545), FoxP3 (clone 236A/E7), and MET (clone 1454Y) (all from Abcam, UK) was performed on formalin-fixed paraffin embedded tissue sections of 9 breast implant-associated anaplastic large cell lymphomas, 16 cutaneous anaplastic large cell lymphomas, 4 ALKnegative anaplastic large cell lymphomas, 4 ALK-positive anaplastic large cell lymphomas, and 5 peripheral T-cell lymphomas, not otherwise specified, and an HRP-labeled polymer detection system was used for visualization (Envision System, Agilent). Additionally, 2 primary effusion lymphoma, 2 Epstein Barr virus (EBV)-positive diffuse large B cell lymphoma, 1 multiple myeloma, and 4 Hodgkin lymphoma $(2 / 4 \mathrm{EBV}+)$ cases were stained for RPS 10 . Breast tissue was used as a positive control for RPS10 based on the protein expression profiles of normal tissues reported by The Human Protein Atlas (http://www.proteinatlas.org/ ENSG00000124614-RPS10/tissue). RPS10, FoxP3, and MET expression was scored in a semiquatitative manner as follows: 0 (negative), 1 (weak), 2 (moderate), 3 (strong). Student's $t$-test was used to compare the expression levels of RPS10, FoxP3 and MET among the different lymphomas.

\section{Results}

\section{Breast implant-associated anaplastic large cell lymphomas have a molecular profile similar to activated CD4 + memory T-cells}

Assuming that lymphoma cells represent aberrant (functional) states of normal T-cell subsets, we applied the deconvolution algorithm CIBERSORT to the breast implant-associated anaplastic large cell lymphoma transcriptional profiles, in order to identify their closest normal counterpart. CIBERSORT is an analytical tool developed to evaluate fractions of normal immune subpopulation in complex tissues using gene expression data. It is based on a 547 gene signature matrix LM22 allowing distinction and enumeration of 22 different immune cell subsets [22]. The CIBERSORT analysis output was consistent with the immunohistochemistry determined immunophenotype of tumor cells, with most breast implant-associated anaplastic large cell lymphomas exhibiting a predominance of activated $\mathrm{CD} 4+$ memory $\mathrm{T}$-cell signatures and the CD4-/CD8 - sample (case 4) showing enrichment for gamma-delta Tcell signatures (Fig. 1A, B).

Subsequently, we compared the CIBERSORT deconvolution output of microdissected breast implant-associated anaplastic large cell lymphoma cells with that obtained from previously generated profiles of other peripheral T-cell lymphomas (including systemic anaplastic large cell lymphomas, angioimmunoblastic T-cell lymphomas and 

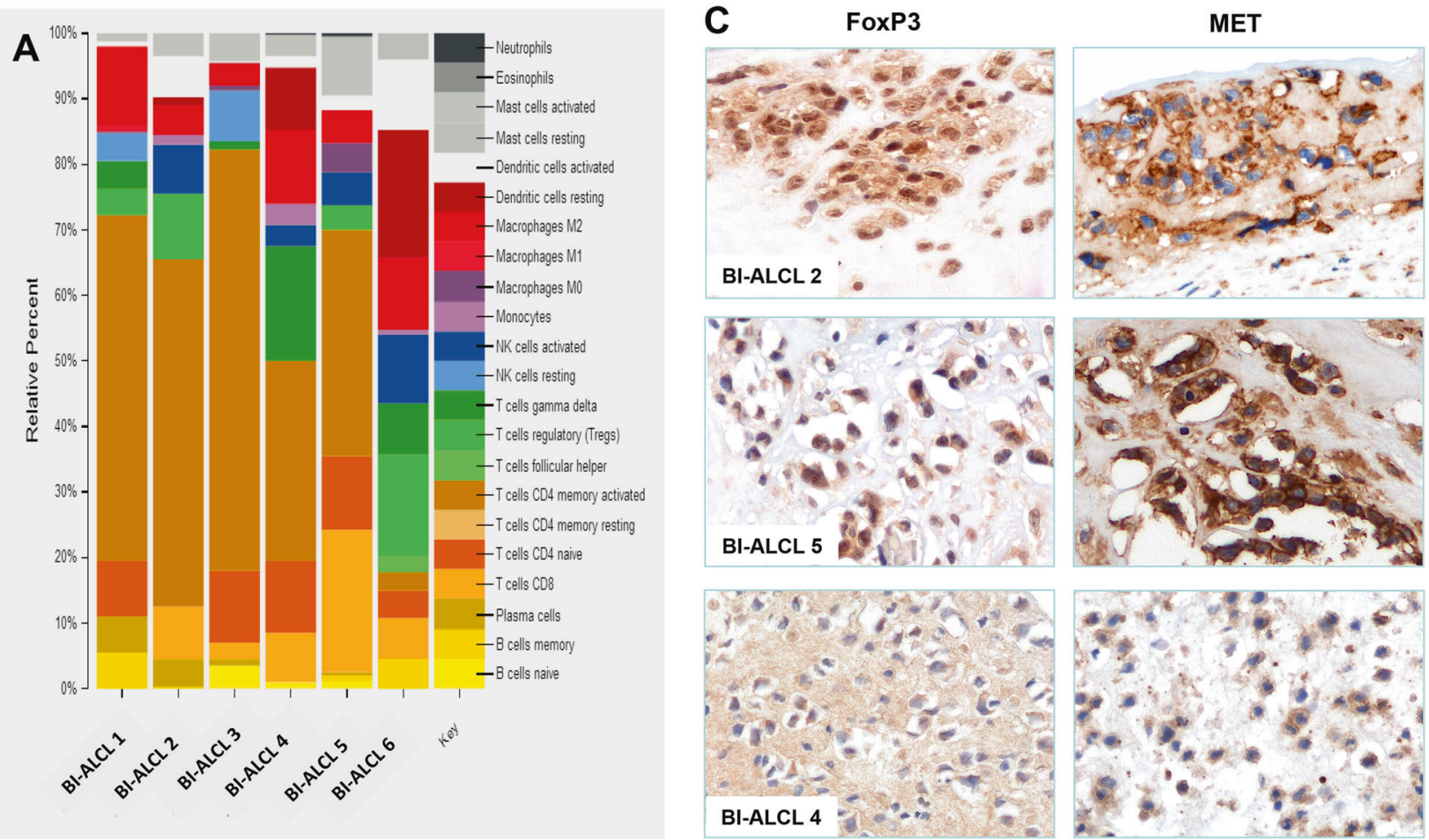

B

Immunohistochemical profile

BI-ALCL 1: ALK-, CD30+, CD15 -/+, CD3-, CD4+, CD8-, GrB+/-, PD1-, IRF4+, CD25+, FoxP3+, MET+

BI-ALCL 2: ALK-, CD30+, CD15 +, CD3+1-, CD4+, CD8-, GrB-, PD1-, IRF4+, CD25+, FoxP3+, MET+

BI-ALCL 3: ALK-, CD30+, CD15-, CD3-, CD4-, CD8+, GrB+, PAX5-, CD25+, FoxP3-, MET-

BI-ALCL 4: ALK-, CD30+, CD15 -, CD3 +/-, CD4-, CD8-, GrB+/-, PAX5-, CD25-, FoxP3-, MET-/+

BI-ALCL 5: ALK-, CD30+, CD15-, CD3-, CD4-, CD8+, GrB+, PD1-, IRF4+/-, CD25+, FoxP3+/-, MET+

BI-ALCL 6: ALK-, CD30+, CD15+, CD3-, CD4+, CD8-, GrB+, PD1-, CD79a+, PAX5-, CD25+, FoxP3+, MET+

Fig. 1 CIBERSORT analysis indicates that breast implant-associated anaplastic large cell lymphomas correspond to activated CD4+ memory T-cells (A). Immunohistochemical profiles of breast implantassociated anaplastic large cell lymphoma (BI-ALCL) samples used for gene expression profiling (B). Strong nuclear FoxP3 staining was noted in all CD4+ CD25+ breast implant-associated anaplastic large cell lymphomas (representative image-BI-ALCL 2), while CD8+ breast implant-associated anaplastic large cell lymphomas showed

peripheral T-cell lymphomas, not otherwise specified) with a high proportion of neoplastic cells. The only significant difference that emerged from the comparison of immune cell signatures between breast implant-associated anaplastic large cell lymphomas and other peripheral T-cell lymphomas was in the fraction of regulatory T-cells, which were overrepresented in breast implant-associated anaplastic large cell lymphomas compared to systemic anaplastic large cell lymphomas (Supplementary Table 1). This finding prompted us to investigate the expression of the regulatory $\mathrm{T}$ cell-related transcription factor FoxP3 in 12 breast implant-associated anaplastic large cell lymphomas, 14 cutaneous anaplastic large cell lymphoma, 4 ALK-positive anaplastic large cell lymphomas, 4 ALK-negative anaplastic large cell lymphomas, and 4 peripheral T-cell lymphomas, absent or variable weak expression (representative image-BI-ALCL 5) and the CD4-CD8 - case (BI-ALCL 4) lacked FoxP3 expression (original magnification $\times 400$ ). MET staining showed strong cytoplasmic and membrane positivity in all except two breast implantassociated anaplastic large cell lymphomas. Only one of the two CD8 + cases expressed MET (BI-ALCL 5) and the CD4-CD8- case showed focal weak expression (BI-ALCL 4) (original magnification $\times 400)(\mathbf{C})$

not otherwise specified by immunohistochemistry (Fig. 5A, C). Along with one peripheral T-cell lymphoma, not otherwise specified, which showed diffuse and intense nuclear staining for Foxp3, only breast implant-associated anaplastic large cell lymphoma cases showed significant FoxP3 expression, especially the 6 breast implantassociated anaplastic large cell lymphoma cases coexpressing CD4 and CD25. The two CD8+ breast implant-associated anaplastic large cell lymphomas, the CD4-/CD8 - case and the three cases with focal or absent CD4 or CD25 expression showed either weak or absent Foxp3 immunoreactivity (Table 1 and Fig. 1C). Since previous studies suggested a $\mathrm{T}$ helper 17/T helper 1-skewed local immune response in implants with capsular fibrosis or breast implant-associated anaplastic large cell lymphomas 
$[24,25]$, we investigated the expression of Th17-related genes IL17A, IL6, TGFBI, and RORCI in breast implantassociated anaplastic large cell lymphomas compared to normal CD4+ T-cells. Of note, the breast implantassociated anaplastic large cell lymphoma cases showed a significant enrichment in RORCI and IL17A transcripts (Supplementary Figure 3). These results suggest either activation-induced FoxP3 expression or a $\mathrm{T}$ helper-like regulatory T-cell status of a proportion of breast implantassociated anaplastic large cell lymphomas.

\section{Breast implant-associated anaplastic large cell lymphomas display deregulated cell motility, downregulation of T-cell receptor signaling, and STAT3 activation}

Supervised comparative analysis of breast implantassociated anaplastic large cell lymphoma and nonneoplastic CD4+ T-cell transcriptional profiles showed 866 differentially expressed genes (Supplementary Table 2). Among these, 310 genes were upregulated and 556 genes were downregulated in breast implant-associated anaplastic large cell lymphoma samples compared to normal T-cells. Based on the expression of these 866 genes, threedimensional principal component analysis showed that anaplastic large cell lymphomas segregated independently from T-cells with the percentage of variance between the groups of $52.3 \%$ (Fig. 2A). Similarly, hierarchical clustering of breast implant-associated anaplastic large cell lymphomas and normal $\mathrm{T}$ cells based on the expression of differentially expressed genes showed a clear separation of the two groups (Fig. 2B). Gene ontology analysis of the differentially expressed transcripts showed overrepresentation of biological processes related to regulation of protein kinase $\mathrm{C}$ activity, cell-matrix adhesion and cell locomotion, and migration and motility in breast implantassociated anaplastic large cell lymphomas. Intriguingly, along with CCR6 and CXCL14, MET and its ligand HGF were among the genes that contributed to the enrichment in cell locomotion and migration and motility programs in breast implant-associated anaplastic large cell lymphomas (Supplementary Table 3). This finding was further investigated in situ by immunohistochemistry for MET protein in 12 breast implant-associated anaplastic large cell lymphomas, 14 cutaneous anaplastic large cell lymphomas, 4 ALKpositive anaplastic large cell lymphomas, 4 ALK-negative anaplastic large cell lymphomas, and 4 peripheral T-cell lymphomas, not otherwise specified (Fig. 5A, C). MET was intensely expressed on the membrane and in the cytoplasm of 10/12 (83\%) breast implant-associated anaplastic large cell lymphomas and weakly and focally in the CD4/CD8double negative breast implant-associated anaplastic large cell lymphoma (case 4), no staining was observed in 1 of the $2 \mathrm{CD} 8+$ breast implant-associated anaplastic large cell lymphoma (case 3) (Fig. 1B). Among the other peripheral T-cell lymphomas, systemic anaplastic large cell lymphomas consistently showed strong membranous and cytoplasmic staining, whereas the cutaneous anaplastic large cell lymphoma and peripheral T-cell lymphoma, not otherwise specified, cases showed more heterogeneity, ranging from strong to no expression (Fig. 5A, C).

Gene set enrichment analysis also identified activation of STAT3 signaling in breast implant-associated anaplastic large cell lymphoma samples when compared to normal Tcells (Fig. 2C). This finding is in accord with the recent descriptions of frequent activating mutations of the $J A K /$ STAT pathway genes in breast implant-associated anaplastic large cell lymphomas $[15,26]$.

The gene ontology biological processes significantly downregulated in breast implant-associated anaplastic large cell lymphomas compared to normal T-cells included viral gene expression-transcription-translation programs related to the downregulation of genes such as EIF3L, EIF3B, EIF3G, and MCTS-1, prototypical inflammatory pathways such as $I L-12$, and $T N F$ and, analogously to what has been observed for systemic ALCLs, T-cell receptor signaling (Supplementary Table 3). The latter finding was further confirmed by gene set enrichment analysis and a molecular classifier based on the expression of genes representative of T-cell receptor signaling, which showed similarities between breast implant-associated anaplastic large cell lymphomas and systemic anaplastic large cell lymphomas (Fig. 2C, D).

\section{The transcriptional profile of breast implant- associated anaplastic large cell lymphoma is distinct from other peripheral T-cell lymphomas}

We next sought to investigate transcriptional differences between breast implant-associated anaplastic large cell lymphomas and other peripheral T-cell lymphomas. First, we conducted unsupervised analysis of breast implantassociated anaplastic large cell lymphomas and systemic ALCLs (systemic anaplastic large cell lymphomas, ALKnegative and ALK-positive). Breast implant-associated anaplastic large cell lymphomas and systemic anaplastic large cell lymphomas appeared distinct by both principal component analysis and hierarchical clustering (Fig. 3A, B). The distinction was also evident on supervised analysis (ANOVA, $p$-value $<0.05$, Bonferroni correction) (Fig. 3C, D), with hierarchical clustering showing a closer relationship of breast implant-associated anaplastic large cell lymphomas with ALK-negative rather than ALK-positive anaplastic large cell lymphomas. The supervised comparison revealed 38 differentially expressed genes between breast implant-associated anaplastic large cell lymphomas 

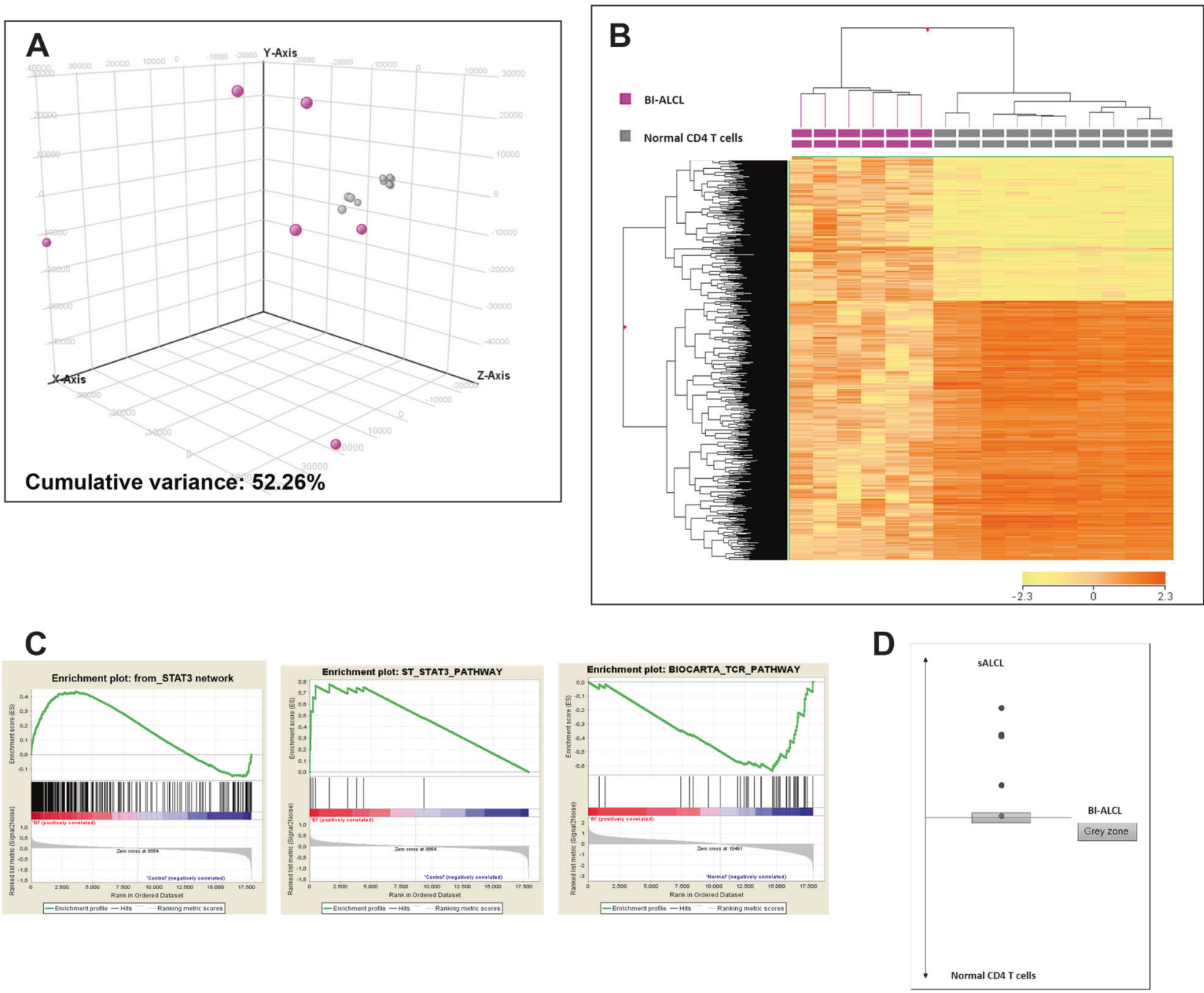

Fig. 2 Supervised analysis of breast implant-associated anaplastic large cell lymphomas (BI-ALCL) vs non-neoplastic CD4+ T-cells. Principal component analysis (A) and hierarchical clustering (B). Gene set enrichment analysis indicates significant enrichment of genes that encode mediators of STAT3 signaling and significant diminution of

T-cell receptor signaling genes (TCR pathway) (C). A molecular classifier based on the expression of genes representative of T-cell receptor signaling showed similarities between breast implantassociated anaplastic large cell lymphomas (BI-ALCL) and systemic anaplastic large cell lymphomas (sALCL) (D)

and systemic anaplastic large cell lymphomas ( $t$-test, $p$ value $<0.05$, fold-change $>2$ ), (Supplementary Table 4), which neatly separated the two groups (Supplementary Figure 4A). Of note, according to the expression of a single gene, RPS10, which emerged as the top ranked gene in this signature $\left(p=1.53 \times 10^{-7}\right.$, fold-change 5.75), the two entities could be discriminated with high accuracy (100\%). A higher number of genes was found to be differentially expressed when breast implant-associated anaplastic large cell lymphomas were compared with either ALK-positive or ALK-negative anaplastic large cell lymphomas. In particular, 317 genes were differently expressed between breast implant-associated anaplastic large cell lymphomas and ALK-positive anaplastic large cell lymphomas (140 genes upregulated and 177 genes downregulated), while 272 genes were differentially expressed between breast implant-

associated anaplastic large cell lymphomas and ALKnegative anaplastic large cell lymphomas (186 genes upregulated and 86 genes downregulated) (Supplementary Tables 5, 6). Gene ontology enrichment analysis revealed that the most overrepresented biological programs in breast implant-associated anaplastic large cell lymphomas as compared to ALK-positive anaplastic large cell lymphomas were related to the signal recognition particle (SRP)-mediated transport of proteins to the endoplasmic reticulum and viral gene expression and transcription (Supplementary Table 7), due to the overexpression of several ribosomal protein genes in breast implant-associated anaplastic large cell lymphomas (e.g., RPS10, RPS19, RPL17, RPL18A). By comparing breast implant-associated anaplastic large cell lymphomas with ALK-negative anaplastic large cell lymphomas, the most overrepresented programs were 

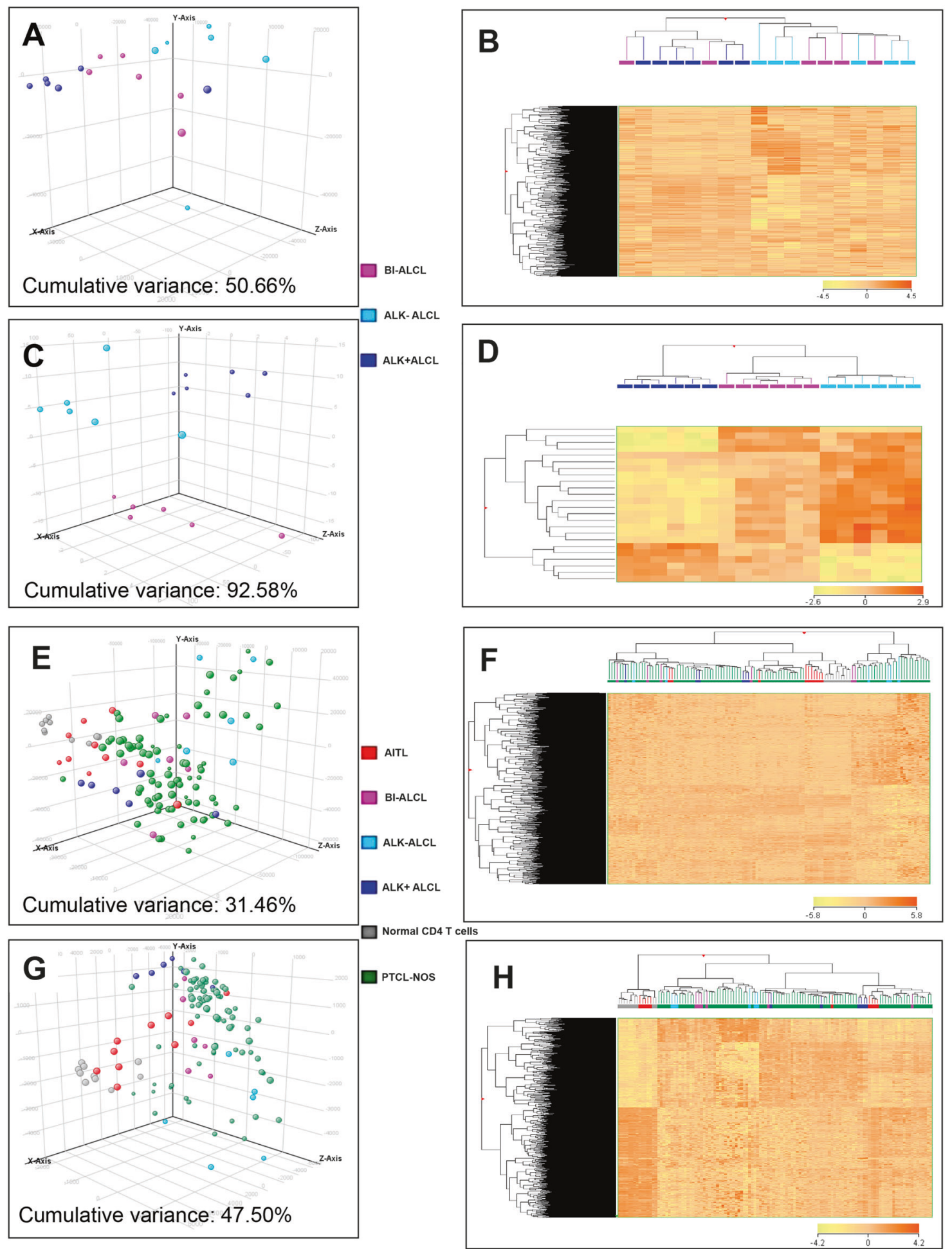

Fig. 3 Breast implant-associated anaplastic large cell lymphoma (BIALCL) is distinct from ALK-positive anaplastic large cell lymphoma (ALK + ALCL) and ALK-negative anaplastic large cell lymphoma (ALK - ALCL) and from peripheral T-cell lymphoma, not otherwise specified (PTCL-NOS) and angioimmunoblastic T-cell lymphoma
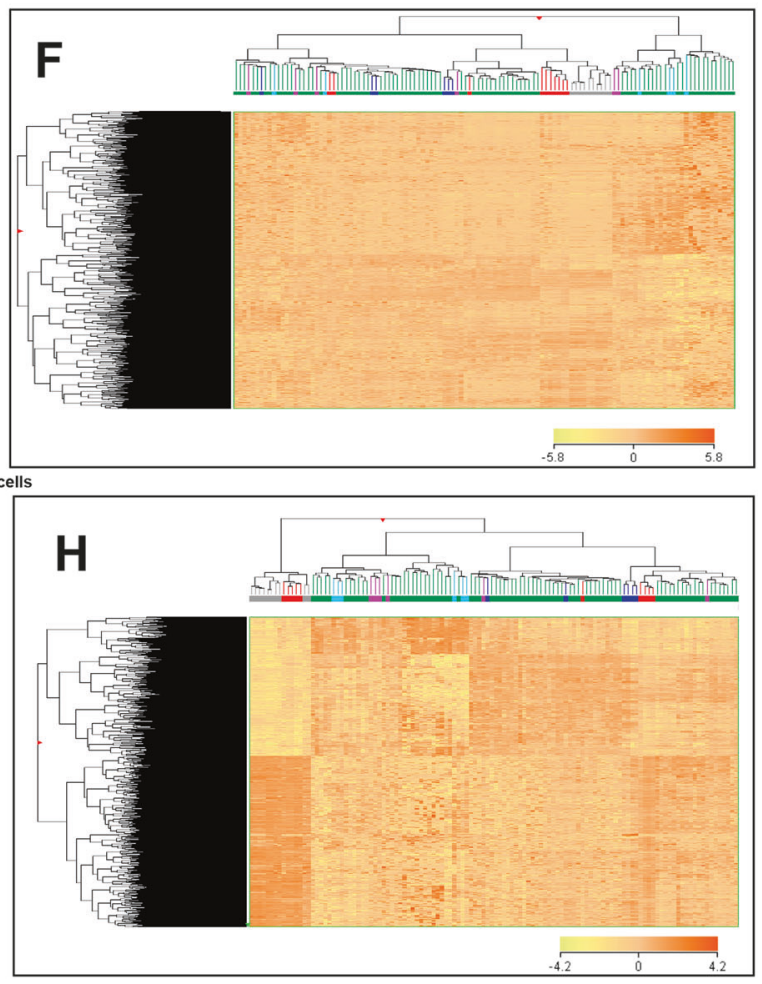

(AITL) based on the global gene expression profiles. Principal component analysis $(\mathbf{A}, \mathbf{C}, \mathbf{E}, \mathbf{G})$ and hierarchical clustering $(\mathbf{B}, \mathbf{D}, \mathbf{F}, \mathbf{H})$ of all entities by cell type. (C, D, G, H One-way ANOVA (Bonferroni) $p$ (Corr) cut-off $=0.05$ )

related to primary microRNA (pri-miRNA) transcripts from a DNA template by RNA polymerase II promoter,

macromolecule metabolic processes, myeloid cell differentiation, and endoplasmic reticulum stress-induced 

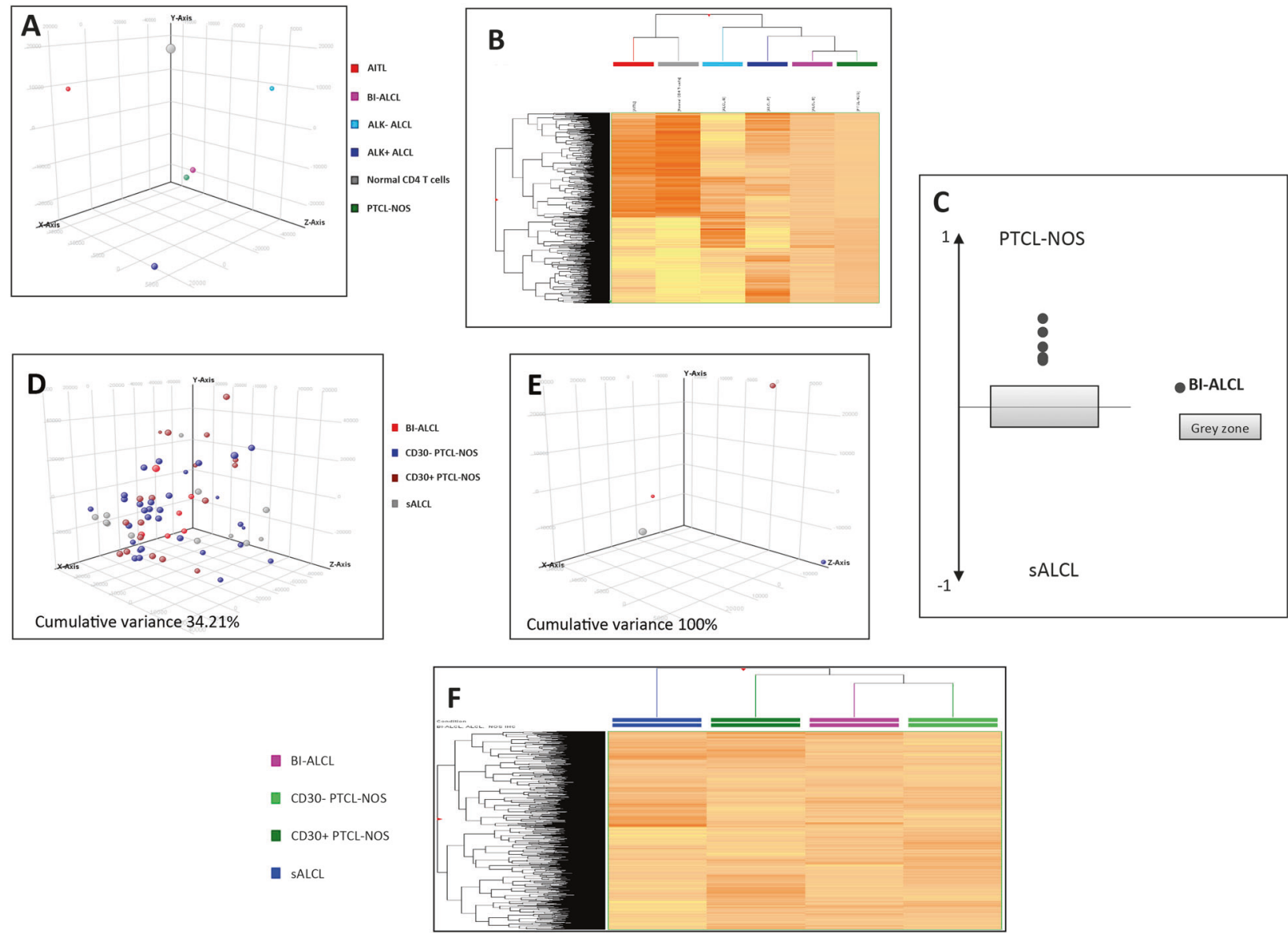

Fig. 4 Relatedness of breast implant-associated anaplastic large cell lymphoma to peripheral T-cell lymphoma subtypes. Breast implantassociated anaplastic large cell lymphoma (BI-ALCL) is closer to peripheral T-cell lymphoma, not otherwise specified (PTCL-NOS), than to ALK-positive anaplastic large cell lymphoma (ALK+ ALCL) and ALK-negative anaplastic large cell lymphoma (ALK- ALCL). Principal component analysis based on the mean expression of all genes (A). Hierarchical clustering (B). A molecular classifier based on the expression of genes discriminating systemic anaplastic large cell lymphoma (sALCL) vs peripheral T-cell lymphoma, not otherwise specified (PTCL-NOS), showed similarities between breast implant-

apoptosis (Supplementary Table 8). No specific gene ontology biological processes could be attributed to downregulated genes in breast implant-associated anaplastic large cell lymphomas as compared to ALK-positive or ALK-negative anaplastic large cell lymphomas.

We then compared the gene expression profiles of breast implant-associated anaplastic large cell lymphomas, systemic anaplastic large cell lymphomas, and other common types of peripheral T-cell lymphomas, including angioimmunoblastic T-cell lymphomas and peripheral T-cell lymphomas, not otherwise specified. These neoplasms could not be distinguished by unsupervised (principal component analysis and hierarchical clustering) (Fig. 3E, F) or supervised (ANOVA, $p$-value $<0.05$, Bonferroni correction) analyses (Fig. 3G, H). However, when cases belonging to each category were grouped (the mean expression being associated anaplastic large cell lymphoma and the latter group (C). Relationship with systemic anaplastic large cell lymphoma (sALCL), CD30 + peripheral T-cell lymphoma, not otherwise specified (CD30+ PTCL-NOS), and CD30 - peripheral T-cell lymphoma, not otherwise specified (CD30- PTCL-NOS). The relationship between breast implant-associated anaplastic large cell lymphoma (BI-ALCL) and peripheral T-cell lymphoma, not otherwise specified, does not appear limited to $\mathrm{CD} 30+$ peripheral T-cell lymphoma, not otherwise specified. Principal component analysis (D), principal component analysis based on the mean expression of all genes (E), and hierarchical clustering (F)

considered for each gene), the different entities could be segregated (Fig. 4A, B). Intriguingly, at the transcriptional level, breast implant-associated anaplastic large cell lymphomas showed an apparent closer relationship to peripheral T-cell lymphomas, not otherwise specified, rather than to anaplastic large cell lymphomas. This observation was confirmed when a classifier able to discriminate between peripheral T-cell lymphomas, not otherwise specified, and systemic anaplastic large cell lymphomas was applied (Fig. 4C). This finding probably reflects the genotypic and phenotypic heterogeneity of peripheral T-cell lymphomas, not otherwise specified, despite no significant difference being observed when $\mathrm{CD} 30+$ and $\mathrm{CD} 30$ - peripheral T-cell lymphoma, not otherwise specified cases (according to immunohistochemistry) were considered separately (Fig. 4D-F). Alternatively, this result might be related to 
differences in the genes that distinguish systemic anaplastic large cell lymphomas from breast implant-associated anaplastic large cell lymphomas and the latter from other peripheral T-cell lymphomas. Indeed, the number of differentially expressed genes between breast implantassociated anaplastic large cell lymphomas and systemic anaplastic large cell lymphomas (i.e., 317 genes vs ALKpositive and 272 genes vs ALK-negative) is higher than between breast implant-associated anaplastic large cell lymphomas and peripheral T-cell lymphomas, not otherwise specified (i.e., 137 genes) (supplementary Figure 4A, B). Among the 137 genes differentially expressed between breast implant-associated anaplastic large cell lymphomas and peripheral T-cell lymphomas, not otherwise specified, 76 were upregulated and 61 were downregulated in breast implant-associated anaplastic large cell lymphomas (Supplementary Table 9). On gene ontology enrichment analysis, downregulated genes (e.g., LCK, ICOS, FYN, CCL19, CCL21, STAT1, CD7) turned out to be significantly involved in specific cellular functions and pathways including T-cell activation and costimulation, cytokinemediated signaling and immune response, whereas upregulated genes (e.g., $H G F, V E G F A, P D G F A$ ) were involved in the regulation of branching structure morphogenesis (Supplemental Table 10). The difference was more pronounced between breast implant-associated anaplastic large cell lymphomas and angioimmunoblastic T-cell lymphomas featuring 1289 differentially expressed genes (608 upregulated and 681 downregulated in breast implant-associated anaplastic large cell lymphoma, Supplementary Table 11). Gene ontology programs associated with genes that were downregulated in breast implant-associated anaplastic large cell lymphomas (e.g., TNFRS18, PDCD2, CD2) were involved in regulation of hemopoiesis, leukocyte activation, apoptotic process, and cytokine production, while the upregulated genes were related to cellular and biological processes (Supplementary Table 12).

Of note, supervised comparative analysis of breast implant-associated anaplastic large cell lymphomas with other peripheral T-cell lymphoma subtypes ( $t$-test, $p$-value $<0.05$, fold-change $>2$ ), identified 5 genes, namely $R N 7 S K$, RPS10, CCR6, ODF2L, and LOC642947 (Supplementary Figure 4B), which were overexpressed in breast implantassociated anaplastic large cell lymphomas. Among these genes, RPS10 was again the most significant one ( $t$-test, $p$ value $<0.0001$, fold-change $>2.7$ ).

Taken together, these data indicate that the molecular profile of breast implant-associated anaplastic large cell lymphomas is different from other peripheral T-cell lymphomas, including systemic anaplastic large cell lymphomas.

\section{RPS10 emerges as a distinguishing marker of breast implant-associated anaplastic large cell lymphoma among peripheral T-cell lymphomas}

To validate the gene transcripts detected by microarray analysis that could discriminate breast implant-associated anaplastic large cell lymphomas from the other types of peripheral T-cell lymphomas, we focused on RPS1O that was part of the 5 gene differentially expressed signature (Supplementary Figure 4B). Real-time quantitative polymerase chain reaction analysis was performed on 11 breast implant-associated anaplastic large cell lymphoma, 4 ALKpositive anaplastic large cell lymphoma, 4 ALK-negative anaplastic large cell lymphoma, and 4 peripheral T-cell lymphoma, not otherwise specified cases. Moreover, 16 cutaneous anaplastic large cell lymphoma samples, for which gene expression profiling data generated by the DASL assay were not available for comparison, were included in the real-time quantitative polymerase chain reaction validation experiment. This analysis confirmed that breast implant-associated anaplastic large cell lymphomas are characterized by a significantly higher mRNA expression of RPS10 compared to other peripheral T-cell lymphomas $(p<0.05)$ (Fig. 5A, B). We then looked for genes whose expression was significantly correlated with that of RPS10 (Pearson correlation $>0.5$ ) in breast implantassociated anaplastic large cell lymphomas, systemic anaplastic large cell lymphomas and peripheral T-cell lymphomas, not otherwise specified. We found 46 genes that were significantly correlated with RPS10. By gene set enrichment analysis, these 46 genes were significantly enriched in specific cellular functions or components comprising RNA metabolism, viral RNA transcription and replication, and the integrin complex (Supplementary Figure 5).

We then investigated whether the increased expression of RPS1O in breast implant-associated anaplastic large cell lymphomas could also be detected at the protein level. To this end, we performed immunohistochemistry for RPS 10 in 11 breast implant-associated anaplastic large cell lymphoma, 14 cutaneous anaplastic large cell lymphoma, 4 ALK-positive anaplastic large cell lymphoma, 4 ALKnegative anaplastic large cell lymphoma, and 4 peripheral T-cell lymphoma, not otherwise specified samples (Fig. 5A, C). The staining intensity was in line with the expression levels of RPS10 detected by real-time quantitative polymerase chain reaction. Breast implant-associated anaplastic large cell lymphoma tumor cells demonstrated strong cytoplasmic RPS10 expression and systemic anaplastic large cell lymphomas and peripheral T-cell lymphomas, not otherwise specified showed weaker or very faint staining, 


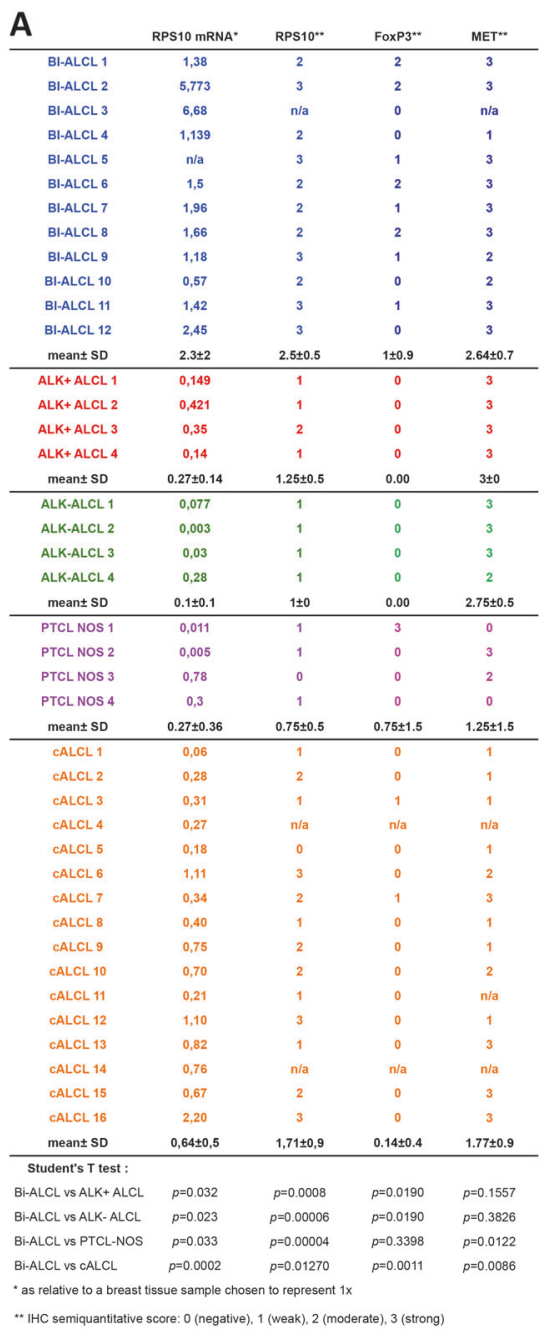

Fig. 5 RPS10, FoxP3 and MET expression in breast implantassociated anaplastic large cell lymphoma (BI-ALCL), cutaneous anaplastic large cell lymphoma (cALCL), peripheral T-cell lymphoma, not otherwise specified (PTCL-NOS), ALK-negative anaplastic large cell lymphoma (ALK - ALCL) and ALK-positive anaplastic large cell lymphoma (ALK+ ALCL) (A). Breast implant-associated anaplastic large cell lymphomas showed the highest RPS10 expression levels among all peripheral T-cell lymphomas by both real-time quantitative polymerase chain reaction $(\mathbf{B})$, and immunohistochemistry $(\mathbf{C}$; original magnification $\times 200$ ). Arrows indicate plasma cells with intense

respectively, while cutaneous anaplastic large cell lymphomas displayed marked heterogeneity, the staining intensity ranging from strong to negative. Among the background cells, an intense reactivity for RPS10 was observed in plasma cells. This finding prompted us to verify the hypothesis that RPS10 expression was associated with high translational activity of either endogenous or viral transcripts. To this end, we assessed RPS10 protein expression in multiple myeloma and in different lymphomas associated with Epstein Barr virus (EBV) or Human Herpes virus-8 (HHV-8) infection, including two HHV-8-positive pleural effusion lymphomas, one peripheral T-cell lymphoma, not otherwise specified with EBV-positive
B

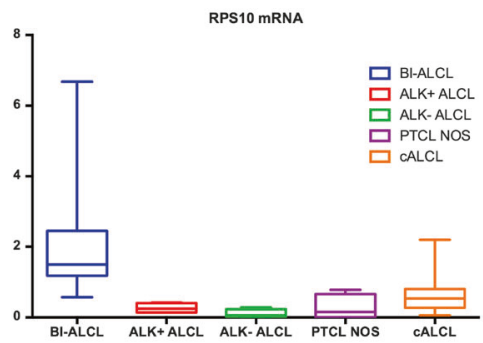

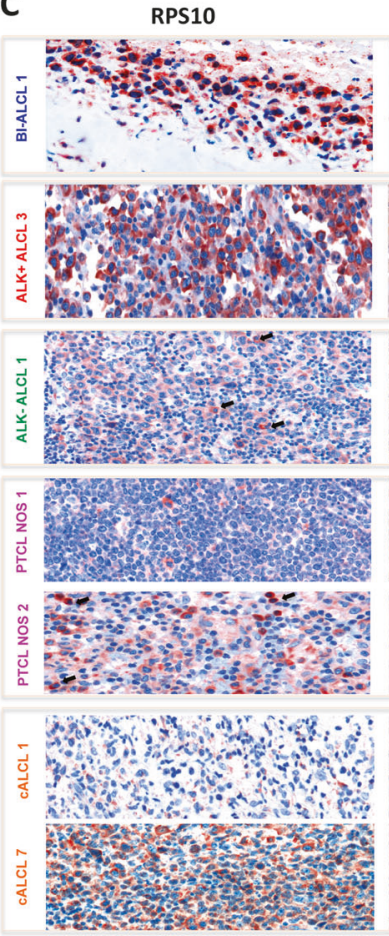

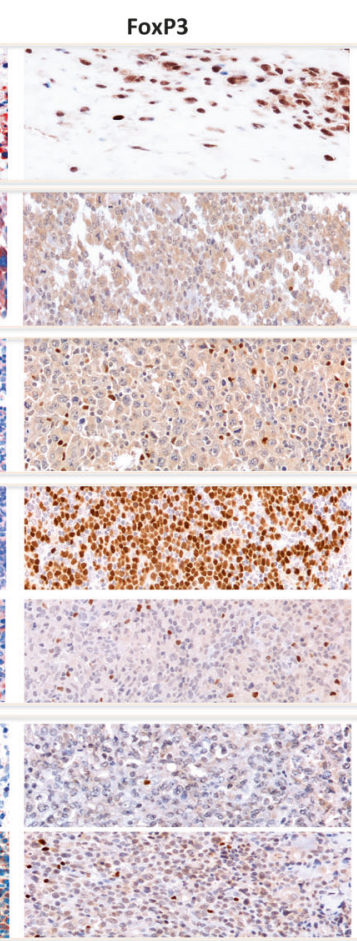

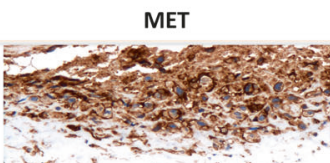
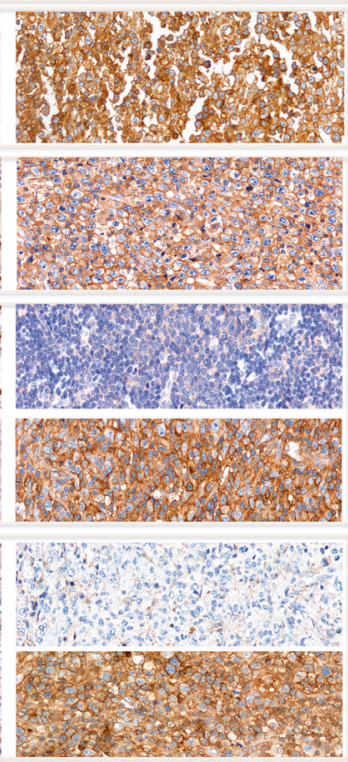

cytoplasmic staining for RPS10. Intense nuclear staining for FoxP3 was mainly observed in breast implant-associated anaplastic large cell lymphomas with the exception of one peripheral T-cell lymphoma, not otherwise specified. The majority of breast implant-associated anaplastic large cell lymphomas, ALK-positive anaplastic large cell lymphomas, and ALK-negative anaplastic large cell lymphomas expressed MET but cases of peripheral T-cell lymphoma, not otherwise specified, and cutaneous anaplastic large cell lymphoma showed variable expression of this receptor tyrosine kinase

Hodgkin/Reed Sternberg-like B-cells, one EBV-positive diffuse large B cell lymphoma, and four Hodgkin lymphomas (2 EBV - and $2 \mathrm{EBV}+$ ). Tumor B-cells from virusinfected lymphomas and multiple myeloma showed intense cytoplasmic positivity, whereas staining was evident only in scattered non-neoplastic plasma cells in the EBV-negative Hodgkin lymphomas (Fig. 6). These findings suggest that increased transcriptional activity, such as that occurring during immunoglobulin production by normal or neoplastic plasma cells or in response to viral infection, might be responsible for the elevated RPS10 expression. A subset of breast implant-associated anaplastic large cell lymphoma samples $(n=4)$ analyzed for EBER, LMP-1, and LANA-1 

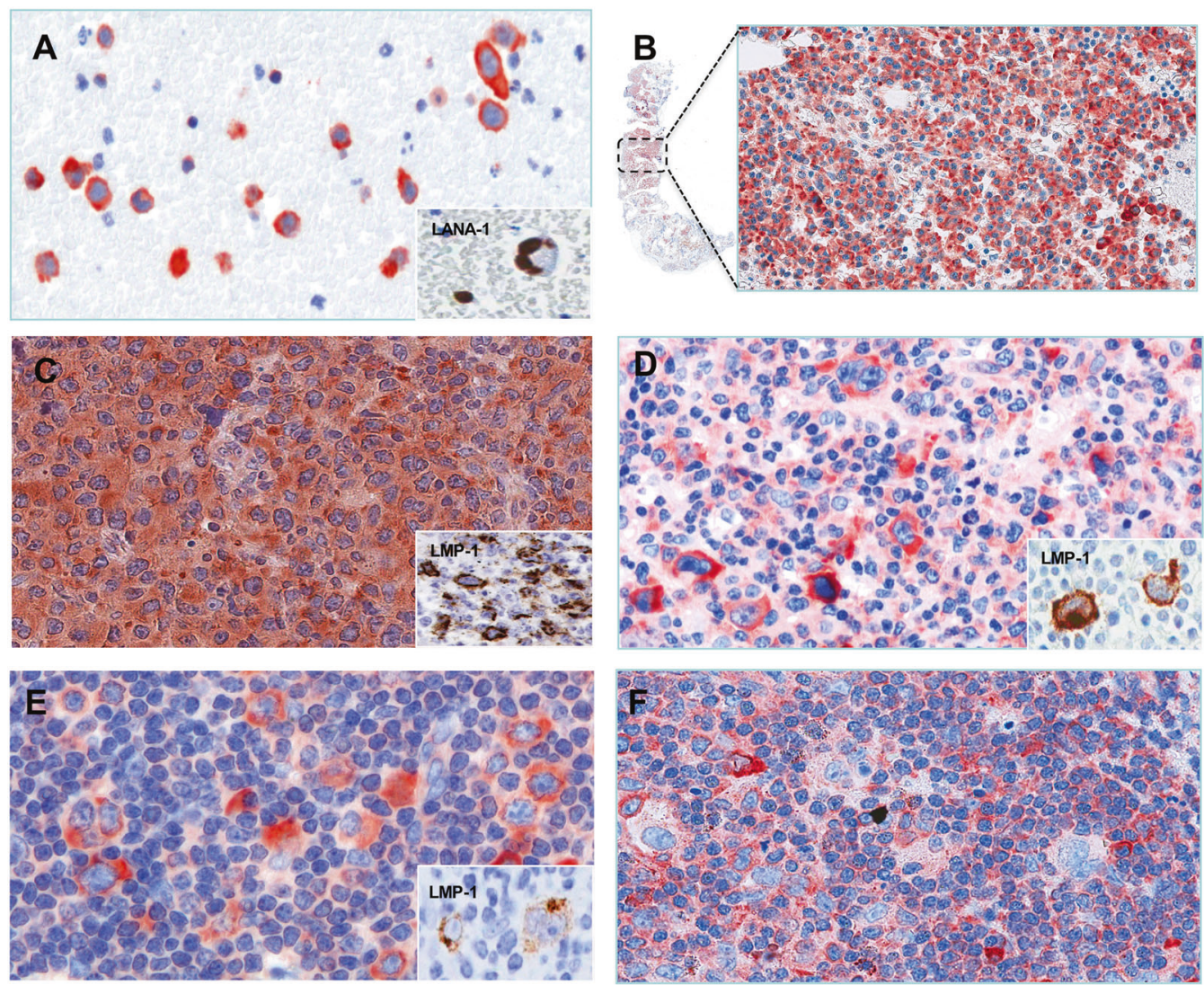

Fig. 6 Intense RPS10 expression in HHV-8+ pleural effusion lymphoma (A), multiple myeloma (B), EBV+ diffuse large B cell lymphoma (C), Hodgkin/Reed Sternberg-like EBV+ B-cells within a peripheral T-cell lymphoma, not otherwise specified (D), and EBV+ Hodgkin lymphoma $(\mathbf{E})$. Weaker staining was observed in EBV-

expression did not shown signs of EBV or HHV-8 infection.

\section{Discussion}

It has been speculated that breast implant-associated anaplastic large cell lymphoma tumor cells may be derived from capsular $\mathrm{T}$ helper 1/T helper 17 cells [25]. Based on our observations, most breast implant-associated anaplastic large cell lymphomas have a transcriptional signature similar to activated CD4+ memory T-cells and display higher levels of RORC1 and IL17A transcripts relative to normal T-cells. In addition, compared to systemic anaplastic large cell lymphomas, breast implant-associated anaplastic large cell lymphomas showed an enrichment in regulatory $\mathrm{T}$ cell-related genes, which was mainly associated with a CD4 $+/ \mathrm{CD} 25+/$ FoxP3 + immunophenotype. It is worth noting that CCL18,CXCL14, and CCR6, which were among the genes upregulated in breast implant-associated anaplastic large cell lymphomas compared to normal T-cells, encode chemokines involved in the migration and differentiation of

negative Hodgkin lymphoma tumor cells $(\mathbf{F})(\mathbf{A}, \mathbf{C}-\mathbf{F}$ original magnification $\times 400$; $\mathbf{B}$ original magnification $\times 10$ and $\times 200$ ). Insets show immunohistochemistry for the HHV-8-encoded protein LANA-1 (inset A; original magnification $\times 400$ ) or for the EBV-encoded protein LMP1 (inset $\mathbf{C}-\mathbf{E}$; original magnification $\times 400$ )

specific leukocyte subsets. In particular, CCR6 is preferentially expressed by immature dendritic cells and memory T-cells (both $\mathrm{T}$ helper 17 and regulatory $\mathrm{T}$ cells) and it has a critical role in cellular migration to inflammatory sites [27, 28]. CCL18 has been reported to be among the most highly expressed chemokines in allergic diseases and it is able to induce conversion of CD4+/CD25memory T-cells to CD4+/CD25+/Foxp3+ regulatory $\mathrm{T}$ cells [29]. CXCL14 mediates a broad spectrum of biological processes, including immune cell migration and antimicrobial immunity [30] and it has recently been shown to play a crucial role in promoting regulatory T-cell activation in stroke [31]. Other genes upregulated in breast implantassociated anaplastic large cell lymphomas that are known to be involved in cell migration and induction of regulatory T-cells were $H G F$ and its receptor $c M E T$. In murine autoimmune models, HGF endows dendritic cells with suppressive ability resulting in effective induction of regulatory T-cells [32]. It has been shown that localization of antigenprimed lymphocytes to antigen-rich non-lymphoid tissue, which is essential for effector-T cell-mediated immunity, is facilitated by the expression of a unique set of "homing" 
receptors acquired by memory T-cells [33, 34]. In this regard, cMET has been shown to instruct T-cell cardiotropism and promote T-cell migration in an experimental heart transplantation model via autocrine chemokine release [35]. Furthermore, HGF-induced chemokinesis requires STAT3 signaling activity [36]. Interestingly, overexpression of cMET has been documented in HTLV-1infected T-cell lines and in adult T-cell leukemia/lymphoma with a liver invasive phenotype [37], both of which have constitutively activated JAK-STAT signaling [38]. Similar to what has been described in Karpas 299 cells and in some other ALCL cell lines [39], we also observed strong membrane expression of cMET in ALK-positive and ALKnegative anaplastic large cell lymphoma cases. It is plausible that cMET expression by breast implant-associated anaplastic large cell lymphoma and other anaplastic large cell lymphoma subtypes might be the consequence of mutational activation of STAT3 [15, 26, 40]. Alternatively, gains of chromosome 7q encompassing the MET locus, which are recurrent aberrations in cutaneous anaplastic large cell lymphomas, could be responsible for its overexpression [41].

Our data suggest that the majority of breast implantassociated anaplastic large cell lymphomas either arise from or acquire the phenotype of activated memory T-cells that are capable of homing to the peri-implant breast capsule. Functional studies are needed in order to clarify whether a proportion of breast implant-associated anaplastic large cell lymphomas derive from Foxp3+/CD25+ regulatory T-cells that collect mainly within the inner layer of non-neoplastic peri-breast implant fibrous capsules [24], acquire a regulatory T-cell program/fate upon neoplastic transformation or upregulate FoxP3 as a consequence of cellular activation, as observed in non-neoplastic T-cells, including memory and effector CD4+ T-cells [42-44]. In keeping with the latter is the hypothesis that breast implant-associated anaplastic large cell lymphomas originate from activated mature cytotoxic $\mathrm{T}$ cells due to the frequent expression of cytotoxic molecules such as Granzyme B $(\mathrm{GrB})$ and T-cell restricted intracellular antigen 1 (TIA1) $[45,46]$.

FoxP3 expression is uncommon in peripheral T-cell lymphomas and until now has mostly been described in adult T-cell leukemia/lymphoma, possibly acquired upon neoplastic conversion of HTLV-1 infected CD4+ T-cells $[47,48]$. Weak expression of FoxP3 has also been observed in ALK-positive anaplastic large cell lymphoma and it has been reported to be induced by NPM/ALK through activation of its key effector STAT3 in this disease $[49,50]$. Similarly, JAK-STAT pathway activation due to mutations or other mechanisms might be responsible for the induction or maintenance of a regulatory $\mathrm{T}$ cell-like phenotype in breast implant-associated anaplastic large cell lymphoma.
Another intriguing hypothesis emerging from our gene expression profiling data is the possible involvement of an infectious agent in the pathogenesis of breast implantassociated anaplastic large cell lymphoma. Signatures related to viral expression, transcription and translation were revealed upon in silico comparison of breast implantassociated anaplastic large cell lymphoma transcriptional profiles with those generated from normal T-cells and ALKpositive anaplastic large cell lymphoma. RPS10 was one of the most differentially expressed genes between breast implant-associated anaplastic large cell lymphoma and other peripheral T-cell lymphomas. It has been shown that RPS10 participates in ribosome biogenesis and in the cellular translational machinery of eukaryotes [51,52] and it also has a crucial role in the formation of the binding site for the internal ribosome entry site (IRES) of several viral transcripts on the human 40S ribosomal subunit [53-55]. The contribution of viral infections to the pathogenesis of specific B- and T-cell lymphomas, including lymphomas presenting as effusions (i.e., primary effusion lymphoma) is well recognized [56]. Our data lead us to speculate that viral infection/re-activation might be a potential trigger of lymphomagenesis besides long-term bacterial infection (i.e., Ralstonia) acquired during implantation, which has been suggested by others to favor the development of breast implant-associated anaplastic large cell lymphoma [57, 58]. This hypothesis, however, requires further investigation. Alternatively, as described for other ribosomal proteins, genetic mutations or external stimuli such as chemical agents, radiation and nutrient depletion could also impair ribosome biogenesis, and result in the accumulation of a ribosome-free form of RPS10 in breast implant-associated anaplastic large cell lymphoma, which may have oncogenic functions [59].

Finally, by comparing the breast implant-associated anaplastic large cell lymphoma transcriptional signature to previously obtained signatures of systemic anaplastic large cell lymphoma, peripheral T-cell lymphoma-not otherwise specified, and angioimmunoblastic T-cell lymphoma, we show that breast implant-associated anaplastic large cell lymphoma represents a distinct subtype of peripheral T-cell lymphoma, not only from a morphological and clinical perspective, but also at the molecular level. As observed in other types of anaplastic large cell lymphomas, breast implant-associated anaplastic large cell lymphoma substantially differs from peripheral T-cell lymphoma, not otherwise specified, with regard to downregulation of antigen-dependent T-cell receptor activation and signaling, which is supported by the frequent T-cell antigen loss observed in most breast implant-associated anaplastic large cell lymphomas [46]. On the other hand, in comparison to ALK-negative anaplastic large cell lymphoma, breast implant-associated anaplastic large cell lymphoma is 
characterized by the upregulation of a myeloid cell differentiation-related program, a finding supported by studies reporting expression of the myeloid antigens CD15, CD13, and CD33 in breast implant-associated anaplastic large cell lymphomas [60, 61], similar to ALK-positive anaplastic large cell lymphomas [62].

In conclusion, in the present study we describe the results of the first comparative transcriptional analysis of breast implant-associated anaplastic large cell lymphoma, which reveals similarities of this neoplasm with activated CD4+ memory $\mathrm{T}$-cells in most cases and differences between breast implant-associated anaplastic large cell lymphoma and other types of peripheral T-cell lymphomas, including systemic anaplastic large cell lymphoma. Functional investigations of the genetic programs and pathways delineated in breast implant-associated anaplastic large cell lymphoma could further advance our understanding of the pathogenesis of this rare malignancy.

\section{Compliance with ethical standards}

Conflict of interest The authors declare that they have no conflict of interest.

\section{References}

1. Di Napoli A, Pepe G, Giarnieri E, et al. Cytological diagnostic features of late breast implant seromas: from reactive to anaplastic large cell lymphoma. PLoS ONE. 2017;12:e0181097.

2. Santanelli di Pompeo F, Laporta R, Sorotos M, et al. Breast implant-associated anaplastic large cell lymphoma: proposal for a monitoring protocol. Plast Reconstr Surg. 2015;136:144e-51e.

3. Nava MB, Adams WP Jr, Botti G, et al. MBN 2016 aesthetic breast meeting BIA-ALCL consensus conference report. Plast Reconstr Surg. 2018;141:40-48.

4. Feldman AL, Harris NL, Stein H, et al. Breast implant-associated anaplastic large cell lymphoma. In: Swerdlow SH, Campo E, Harris NL, et al. editors. WHO classification of tumours of haematopoietic and lymphoid tissues. World Health Organization Classification of Tumours. Revised. 4th ed. Lyon, France: International Agency of Research on Cancer, 2017. p. 421-2.

5. Clemens MW, Medeiros LJ, Butler CE, et al. Complete surgical excision is essential for the management of patients with breast implant-associated anaplastic large-cell lymphoma. J Clin Oncol. 2016;34:160-8.

6. Morris SW, Kirstein MN, Valentine MB, et al. Fusion of a kinase gene, ALK, to a nucleolar protein gene, NPM, in non-Hodgkin's lymphoma. Science. 1994;263:1281-4.

7. Savage KJ, Harris NL, Vose JM, et al. ALK- anaplastic large-cell lymphoma is clinically and immunophenotypically different from both ALK+ALCL and peripheral T-cell lymphoma, not otherwise specified: report from the International Peripheral T-Cell Lymphoma Project. Blood. 2008;111:5496-504.

8. Kempf W, Pfaltz K, Vermeer MH, et al. EORTC, ISCL, and USCLC consensus recommendations for the treatment of primary cutaneous CD30-positive lymphoproliferative disorders: lymphomatoid papulosis and primary cutaneous anaplastic large-cell lymphoma. Blood. 2011;118:4024-35.
9. Bizjak M, Selmi C, Praprotnik S, et al. Silicone implants and lymphoma: The role of inflammation. J Autoimmun. 2015;65:64-73.

10. Thompson MA, Stumph J, Henrickson SE, et al. Differential gene expression in anaplastic lymphoma kinase-positive and anaplastic lymphoma kinase-negative anaplastic large cell lymphomas. Hum Pathol. 2005;36:494-504.

11. Piva R, Agnelli L, Pellegrino E, et al. Gene expression profiling uncovers molecular classifiers for the recognition of anaplastic large-cell lymphoma within peripheral T-cell neoplasms. J Clin Oncol. 2010;28:1583-90.

12. Piccaluga PP, Fuligni F, De Leo A, et al. Molecular profiling improves classification and prognostication of nodal peripheral Tcell lymphomas: results of a phase III diagnostic accuracy study. J Clin Oncol. 2013;31:3019-25.

13. de Leval L, Rickman DS, Thielen C, et al. The gene expression profile of nodal peripheral T-cell lymphoma demonstrates a molecular link between angioimmunoblastic T-cell lymphoma (AITL) and follicular helper $\mathrm{T}$ (TFH) cells. Blood. 2007;109:4952-63.

14. Bisig B, de Reynies A, Bonnet C, et al. CD30-positive peripheral T-cell lymphomas share molecular and phenotypic features. Haematologica. 2013;98:1250-8.

15. Di Napoli A, Jain P, Duranti E, et al. Targeted next generation sequencing of breast implant-associated anaplastic large cell lymphoma reveals mutations in JAK/STAT signalling pathway genes, TP53 and DNMT3A. Br J Haematol. 2018;180:741-4.

16. Orofino N, Guidotti F, Cattaneo D, et al. Marked eosinophilia as initial presentation of breast implant-associated anaplastic large cell lymphoma. Leuk Lymphoma. 2016;57:2712-5.

17. Di Napoli A, Giubettini M, Duranti E, et al. Iatrogenic EBVpositive lymphoproliferative disorder with features of EBV +mucocutaneous ulcer: evidence for concomitant TCR $\gamma / \mathrm{IGH}$ rearrangements in the Hodgkin-like neoplastic cells. Virchows Arch. 2011;458:631-6.

18. Ritchie ME, Phipson B, Wu D, et al. Limma powers differential expression analyses for RNA-sequencing and microarray studies. Nucleic Acids Res. 2015;43:e47.

19. Johnson WE, Li C, Rabinovic A. Adjusting batch effects in microarray expression data using empirical Bayes methods. Biostatistics. 2007;8:118-27.

20. Navari M, Fuligni F, Laginestra MA, et al. Molecular signature of Epstein Barr virus-positive Burkitt lymphoma and post-transplant lymphoproliferative disorder suggest different roles for Epstein Barr virus. Front Microbiol. 2014;5:728.

21. Floratos A, Smith K, Ji Z, Watkinson J, Califano A. geWorkbench: an open source platform for integrative genomics. Bioinformatics. 2010;26:1779-80.

22. Newman AM, Liu CL, Green MR, et al. Robust enumeration of cell subsets from tissue expression profiles. Nat Methods. 2015;12:453-7.

23. Livak KJ, Schmittgen DT. Analysis of relative gene expression data using real-time quantitative PCR and the 2(-Delta Delta C(T)) Method. Methods. 2001;25:402-8.

24. Wolfram D, Rabensteiner E, Grundtman C, et al. T regulatory cells and TH17 cells in peri-silicone implant capsular fibrosis. Plast Reconstr Surg. 2012;129:327e-37e.

25. Kadin ME, Deva A, Xu H, et al. Biomarkers provide clues to early events in the pathogenesis of breast implant-associated anaplastic large cell lymphoma. Aesthetic Surg J. 2016;36:773-81.

26. Blombery $\mathrm{P}$, Thompson E, Jones $\mathrm{K}$, et al. Whole exome sequencing reveals activating JAK1 and STAT3 mutations in breast implant-associated anaplastic large cell lymphoma anaplastic large cell. Haematologica. 2016;101:e387-e390.

27. Ebert LM, McColl SR. Up-regulation of CCR5 and CCR6 on distinct subpopulations of antigen-activated CD4+T lymphocytes. J Immunol. 2002;168:65-72. 
28. Kleinewietfeld M, Puentes F, Borsellino G, Battistini L, Rötzschke O, Falk K. CCR6 expression defines regulatory effector/memory-like cells within the CD25(+)CD4+T-cell subset. Blood. 2005;105:2877-86.

29. Chang Y, de Nadai P, Azzaoui I, et al. The chemokine CCL18 generates adaptive regulatory $\mathrm{T}$ cells from memory $\mathrm{CD} 4+\mathrm{T}$ cells of healthy but not allergic subjects. FASEB J. 2010;24: 5063-72.

30. Lu Jing, Chatterjee Mita, Schmid Hannes, Beck Sandra, Gawaz Meinrad. CXCL14 as an emerging immune and inflammatory modulator. J Inflamm. 2016;13:1.

31. Lee HT, Liu SP, Lin CH, et al. A crucial role of CXCL14 for promoting regulatory $\mathrm{T}$ cells activation in stroke. Theranostics. 2017;7:855-75.

32. Benkhoucha M, Santiago-Raber ML, Schneiter G, et al. Hepatocyte growth factor inhibits CNS autoimmunity by inducing tolerogenic dendritic cells and CD25+Foxp3+regulatory $\mathrm{T}$ cells. Proc Natl Acad Sci USA. 2010;107:6424-9.

33. McCully ML, Ladell K, Hakobyan S, Mansel RE, Price DA, Moser B. Epidermis instructs skin homing receptor expression in human T cells. Blood. 2012;120:4591-8.

34. Mora JR, Von Andrian UH. T-cell homing specificity and plasticity: new concepts and future challenges. Trends Immunol. 2006;27:235-43.

35. Komarowska I, Coe D, Wang G, et al. Hepatocyte growth factor receptor c-Met instructs $\mathrm{T}$ cell cardiotropism and promotes $\mathrm{T}$ cell migration to the heart via autocrine chemokine release. Immunity. 2015;42:1087-99.

36. Khabbazi S, Jacques RO, Moyano Cardaba C, Mueller A. Janus kinase 2 and signal transducer and activator of transcription 3 activation is not essential for CCL3-, CCL5- or CCL8-induced chemotaxis. Cell Biochem Funct. 2013;31:312-8.

37. Imaizumi Y, Murota H, Kanda S, et al. Expression of the c-Met proto-oncogene and its possible involvement in liver invasion in adult T-cell leukemia. Clin Cancer Res. 2003;9:181-7.

38. Migone TS, Lin JX, Cereseto A, Mulloy JC, et al. Constitutively activated Jak-STAT pathway in T cells transformed with HTLV-I. Science. 1995;269:79-81.

39. Crescenzo R, Abate F, Lasorsa E, et al. Convergent mutations and kinase fusions lead to oncogenic STAT3 activation in anaplastic large cell lymphoma. Cancer Cell. 2015;27:516-32.

40. Christensen JG, Zou HY, Arango ME, et al. Cytoreductive antitumor activity of PF- 2341066, a novel inhibitor of anaplastic lymphoma kinase and c-Met, in experimental models of anaplastic large-cell lymphoma. Mol Cancer Ther. 2007;6:3314-22.

41. van Kester MS, Tensen CP, Vermeer MH, et al. Cutaneous anaplastic large cell lymphoma and peripheral T-cell lymphoma NOS show distinct chromosomal alterations and differential expression of chemokine receptors and apoptosis regulators. J Invest Dermatol. 2010;130:563-75.

42. Ziegler SF. FOXP3: not just for regulatory T cells anymore. Eur J Immunol. 2007;37:21-23.

43. Halim L, Romano M, McGregor R, et al. An atlas of human regulatory $\mathrm{T}$ helper-like cells reveals features of Th2-like Tregs that support a tumorigenic environment. Cell Rep. 2017;20:757-70.

44. Kitz A1, Dominguez-Villar M2. Molecular mechanisms underlying Th1-like Treg generation and function. Cell Mol Life Sci. 2017;74:4059-75.
45. Xu J, Wei S. Breast implant-associated anaplastic large cell lymphoma: review of a distinct clinicopathologic entity. Arch Pathol Lab Med. 2014;138:842-6.

46. Laurent C, Delas A, Gaulard P, et al. Breast implant-associated anaplastic large cell lymphoma: two distinct clinicopathological variants with different outcomes. Ann Oncol. 2016;27:306-14.

47. Roncador G, Garcia JF, Garcia JF, et al. FOXP3, a selective marker for a subset of adult T-cell leukaemia/lymphoma. Leukemia. 2005;19:2247-53.

48. Walsh PT, Benoit BM, Wysocka M, et al. A role for regulatory $\mathrm{T}$ cells in cutaneous T-cell lymphoma: induction of a CD4+CD25 +Foxp3+T-cell phenotype associated with HTLV-1 infection. J Invest Dermatol. 2006;126:690-2.

49. Bonzheim I, Geissinger E, Tinguely M, et al. Evaluation of FoxP3 expression in peripheral T-cell lymphoma. Am J Clin Pathol. 2008;130:613-9.

50. Kasprzycka M, Marzec M, Liu X, Zhang Q, Wasik MA. Nucleophosmin/anaplastic lymphoma kinase (NPM/ALK) oncoprotein induces the $\mathrm{T}$ regulatory cell phenotype by activating STAT3. PNAS. 2006;103:9964-9.

51. Ren J, Wang Y, Liang Y, Zhang Y, Bao S, Xu Z. Methylation of ribosomal protein $\mathrm{S} 10$ by protein-arginine methyltransferase 5 regulates ribosome biogenesis. J Biol Chem. 2010;285:12695-705.

52. Warner JR, McIntosh KB. How common are extraribosomal functions of ribosomal proteins? Mol Cell. 2009;34:3-11.

53. Malygin AA, Shatsky IN, Karpova GG. Proteins of the human $40 \mathrm{~S}$ ribosomal subunit involved in hepatitis C IRES binding as revealed from fluorescent labeling. Biochemistry. 2013;78:53-59.

54. Kang ST, Wang HC, Yang YT, Kou GH, Lo CF. The DNA virus white spot syndrome virus uses an internal ribosome entry site for translation of the highly expressed nonstructural protein ICP35. J Virol. 2013;87:13263-78.

55. Abbas W1, Dichamp I, Herbein G. The HIV-1 Nef protein interacts with two components of the $40 \mathrm{~S}$ small ribosomal subunit, the RPS10 protein and the 18S rRNA. Virol J. 2012;9:103.

56. Jaffe ES, Campo E, Harris NL, et al. Introduction and overview of the classification of lymphoid neoplasms. In: Swerdlow SH, Campo E, Harris NL, et al., editors. WHO classification of tumours of haematopoietic and lymphoid tissues. World Health Organization Classification of Tumours. Revised 4th ed. Lyon, France: International Agency of Research on Cancer, 2017. p. 190-8.

57. Deva AK. Commentary on: CD30+T cells in late seroma may not be diagnostic of breast implant-associated anaplastic large cell lymphoma. Aesthet Surg J. 2017;37:779-81.

58. Hu H, Johani K, Almatroudi A, et al. Bacterial biofilm infection detected in breast implant-associated anaplastic large-cell lymphoma. Plast Reconstr Surg. 2016;137:1659-69.

59. Zhou X, Liao WJ, Liao JM, Liao P, Lu H. Ribosomal proteins: functions beyond the ribosome. J Mol Cell Biol. 2015;7:92-104.

60. Wu D, Allen CT, Fromm JR. Flowcytometry of ALK-negative anaplastic large cell lymphoma of breast implant-associated effusion and capsular tissue. Cytom B Clin Cytom. 2015;88:58-63.

61. Montgomery-Goecker C, Fuda F, Krueger JE, Chen W. Immunophenotypic characteristics of breast implant-associated anaplastic large-cell lymphoma by flowcytometry. Cytom B Clin Cytom. 2015;88:291-3.

62. Juco J, Holden JT, Mann KP, Kelley LG, Li S. Immunophenotypic analysis of anaplastic large cell lymphoma by flow cytometry. Am J Clin Pathol. 2003;119:205-12. 